\title{
The Management of Breast Symptoms in the Pregnant and Lactating Patient
}

\author{
Sheila S. Lee • Heidi J. Hartman • Cherie M. Kuzmiak • \\ Kenneth L. Crosby
}

Published online: 5 January 2013

(C) Springer Science+Business Media New York 2013

\begin{abstract}
With a trend toward childbearing at more advanced ages, the incidence of pregnancy-associated breast cancer is expected to increase. Nevertheless, the majority of breast disorders in pregnant patients are the same as those in nonpregnant patients, with a few unique exceptions. Although many of the presenting symptoms are similar, the physiologic changes of pregnancy pose unique diagnostic challenges for the obstetrician, gynecologist, and radiologist, which may contribute to a delay in diagnosis of breast cancer. Awareness of benign and malignant breast disorders and a familiarity with current recommendations for the diagnostic imaging evaluation of breast symptoms in the pregnant patient may aid in earlier detection of pregnancy-associated breast cancer and improve outcomes for these women.
\end{abstract}

Keywords Breast diseases $\cdot$ PABC (pregnancy associated breast carcinoma) $\cdot$ Pregnancy $\cdot$ Lactation $\cdot$ Ultrasound · Mammography $\cdot$ Radiology

\section{Introduction}

Annual screening mammography is recommended for asymptomatic women beginning at age 40 years or sooner if they are high risk for developing breast cancer [1]. Pregnant patients,

S. S. Lee $(\varangle) \cdot$ H. J. Hartman • C. M. Kuzmiak $\cdot$ K. L. Crosby Department of Radiology, University of North Carolina, CB\#7510, Physicians Office Building, Rm \#116, 170 Manning Drive, Chapel Hill, NC 27599, USA

e-mail: sheila_lee@med.unc.edu

H. J. Hartman

e-mail: Heidi_hartman@med.unc.edu

C. M. Kuzmiak

e-mail: Cherie_kuzmiak@med.unc.edu

K. L. Crosby

e-mail: Kenneth_crosby@med.unc.edu however, are typically younger than age 40 years and not yet of screening age. Thus, a diagnosis of breast disease in these patients is usually not made from a mammographic finding but rather a physical finding, such as a mass, skin changes, nipple discharge, or lymphadenopathy $[2,3]$.

Although the majority of breast disorders diagnosed during pregnancy are benign, it is estimated that approximately 1 in 3,000 pregnancies are complicated by breast cancer [4]. Pregnancy-associated breast cancer (PABC) is defined as breast cancer found either during pregnancy or during the first year after pregnancy. During the past several decades, there has been an increase in the incidence of PABC [5]. With the trend toward advanced childbearing ages, the incidence of PABC is suspected to increase further [6, 7]. Invasive ductal carcinoma is the most common type of invasive breast cancer to occur in these patients. A few other cancers have been reported but are significantly less frequent [3, 8-10]. In cases of PABC, more than $50 \%$ of patients have high-grade tumors, and more than $50 \%$ of patients present with lymph node involvement at the time of diagnosis $[8,10]$. Because of a more aggressive tumor and the delay in diagnosis, these patients present with advanced stages of disease and have a poorer outcome than do women of the same age with breast cancer [3, 8-10]. Recurrences are common and usually appear within 2-3 years of initial diagnosis [8].

Understanding the effect of pregnancy on various breast disorders, the management of common presenting symptoms, and the diagnostic imaging algorithm for these symptoms during pregnancy may reduce the delay of diagnosis of PABC.

\section{Clinical Evaluation}

Physiologic changes of the breast, occurring in response to rising hormone levels during pregnancy, result in increased volume and firmness of the breast and diffusely increased 
parenchymal density. As a result, the physical examination of the gravid breast can be challenging. Nevertheless, physical examination of a symptom in this patient population is important and can direct the imaging evaluation.

\section{Masses}

The majority of breast masses exist before pregnancy but may present as an enlarging or newly painful mass as they respond to pregnancy-associated hormonal changes. Approximately $90 \%$ of patients with PABC present with a palpable mass $[2,3,7,8]$. Less frequently, breast cancer will present as breast erythema, breast swelling or enlargement, bloody nipple discharge, or local or distant metastasis. Robbins et al. evaluated 134 lesions in 126 pregnant or postpartum women of which $89 \%$ presented with a symptomatic lesion (i.e., palpable finding, nipple discharge, or pain). The remaining asymptomatic lesions included: a) lesions classified as probably benign according to the American College of Radiology Breast Imaging Reporting and Data System (BI-RADS), which were being followed after their initial detection on screening mammogram performed prior to pregnancy; $b$ ) lesions called back from screening mammograms performed during lactation; or c) lesions from routine surveillance imaging in patients with a prior history of breast cancer. A palpable mass was the presenting symptom in the majority of patients $(64 \%)$ followed by erythema $(8 \%)$, pain $(5 \%)$, thickening ( $3 \%)$, bloody nipple discharge $(2 \%)$, nonbloody nipple discharge $(1 \%)$, milk rejection (1\%), breast firmness (1\%), or dimpling (7\%) [2]. In another study, Ahn et al. evaluated 22 patients with PABC. Of those, $81.8 \%$ presented with a palpable mass. Notably, $18.2 \%$ of those patients diagnosed with PABC presented with unilateral breast enlargement and thickening. Furthermore, in $13 \%$, palpable lymphadenopathy was detected along with the breast finding [3].

Benign masses found in both pregnant and nonpregnant women include cysts, fibroadenomas, phyllodes tumors, papillomas, and fat necrosis. Lactating adenomas and galactoceles are unique to the pregnant or lactating breast.

Cysts occur in $10 \%$ of all women. They can produce a palpable lump, can be painful, and can grow and regress rapidly. Mammographically, they appear as hypo- to isodense round or oval circumscribed masses. On ultrasound, they are anechoic masses with imperceptible walls and posterior acoustic enhancement. Internal echogenicity can be interpreted as debris if visualized floating in real time. A complicated cyst, with internal proteinaceous material or blood, is difficult to distinguish from a solid mass and should be aspirated. Painful cysts also can be aspirated under ultrasound-guidance [11].

Fibroadenomas, which contain stromal tissue and structures suggesting breast ductules, are the most common breast mass presenting in women younger than 35 years. On mammogram, they appear as an isodense, oval, or lobulated mass that may or may not have associated course calcifications. A homogenous, hypoechoic, oval, or lobular solid mass, sometimes with posterior acoustic enhancement, is usually seen on ultrasound. It is oriented parallel to the chest wall. Fibroadenomas may develop or markedly enlarge during pregnancy in response to increased estrogen and can regress in the postpartum period. Their appearance in pregnant or lactating patients is no different than in nonpregnant patients, although a complex appearance with cystic spaces and prominent ducts can be seen.

Biopsy is recommended for fibroadenomas that are newly palpable or increasing in size or those demonstrating suspicious features [11]. Biopsy-proven fibroadenomas require only clinical follow-up to ensure stability or regression in patients who are not yet receiving annual screening mammography $[8,11]$. At our institution, biopsy-proven fibroadenomas that are considered radiographically concordant undergo 1-year follow-up in the over-40 or screening population. Several articles advocate that fibroadenomas may be managed with imaging surveillance as opposed to biopsy if there are only benign features on initial imaging and they are nonpalpable [12-14].

Lactating adenomas are benign stromal tumors that may represent a variant of fibroadenomas, tubular adenomas, or lobular hyperplasia. They are seen from the third trimester through lactation, and their natural course is regression following the cessation of breast feeding. The sonographic appearance is typically a hypoechoic oval or lobulated mass that can be difficult to distinguish from the surrounding breast parenchyma.

Spontaneous infarction may develop within fibroadenomas and lactating adenomas, usually during the third trimester or after delivery. Clinically, this phenomenon presents as the sudden onset of pain in a previously painless fibroadenoma or lactating adenoma. Infarction can lead to a change in the radiographic appearance of the mass with suspicious findings that require core biopsy for definitive diagnosis [8, 11, 15-17].

Histologically similar to fibroadenoma, a phyllodes tumor usually presents as a rapidly enlarging mass. Although there does not appear to be evidence that phyllodes tumors are hormone receptive, they can rarely present as a rapidly enlarging mass in the pregnant, as well as the nonpregnant patient [18]. The mammographic and sonographic appearance is similar to a fibroadenoma, although a complex appearance and cystic spaces can also be seen. Approximately $10 \%$ of phyllodes tumors are malignant [19] with no reliable imaging characteristics to distinguish them from the benign form [11]. There is a low incidence of lung, bone, and liver metastasis. Because benign phyllodes tumors are difficult to distinguish pathologically from malignant ones, the recommended treatment is surgical excision with clear margins to avoid local recurrence [11]. 
Intraductal papillomas can occur as solitary or multiple lesions. If solitary, they are typically subareolar, and if multiple they are typically peripheral. The classic clinical symptom is bloody nipple discharge, but they also can cause spontaneous clear discharge. On mammogram, they are usually round or oval, circumscribed, isodense masses that can contain calcifications. On ultrasound, papillomas appear as solid, round or oval, hypoechoic masses, sometimes within a fluid-filled duct. Papillomas produce an intraductal filling defect on MRI and ductography. Treatment is usually surgical excision to exclude the presence of malignancy, although follow-up for papillomas diagnosed by core biopsy is controversial.

Historically, benign papillomas were recommended for surgical excision because of the difficulty to distinguish benign from malignant papillary lesions at pathology. Based on our experience, benign intraductal papillomas diagnosed on core needle biopsy with radiology and pathology concordance may be managed with imaging follow-up rather than surgical excision. Papillary lesions with atypia or classified as complex on core needle biopsy, however, should be surgically excised because of a $6.9 \%$ histologic underestimation of cancer (Kuzmiak, unpublished data).

It should be noted that juvenile papillomatosis can be considered a potential risk factor for breast cancer given its association with carcinoma in up to $15 \%$ of cases, as well as in nearly $50 \%$ of female relatives, for whom follow-up also is mandatory $[8,11]$.

Fat necrosis is a benign condition that is commonly the result of trauma of which the patient may or may not be aware. An oil cyst associated with fat necrosis can present as a firm, palpable mass. On mammogram, an oil cyst is characteristically a lucent round circumscribed mass that may exhibit a thin rim of calcification. Its appearance at ultrasound is characteristically an anechoic, circumscribed mass with through transmission at the palpable site and is considered benign. If there is rim calcification of the oil cyst, then there may be a conspicuous smooth curvilinear shadowing originating from the top of the mass, obscuring the inferior portion of the mass. Occasionally, fat necrosis can present as an irregular mass sonographically with indistinct margins with or without an echogenic halo. In these instances, malignancy cannot be excluded. If fat necrosis is suspected based on a history of trauma or ecchymosis at the site, a mammogram may be helpful to confirm the presence of fat or oil cysts to avoid a core needle biopsy. If there is any uncertainty about the precise site of the inciting trauma, biopsy should be performed to exclude malignancy.

Galactoceles are the result of an obstructed milk duct and occur both during and after cessation of lactation. Although the mammographic and sonographic appearance is dependent on fat and water content, a fat-fluid level is a diagnostic sign that can be seen on a 90-degree true lateral mammogram, as well as ultrasound. Additional sonographic features include a round or oval, smooth-walled structure with variable internal echogenicity. Most galactoceles regress over time; however, ultrasound-guided needle aspiration can be both therapeutic and diagnostic, yielding fluid milk $[8,11,17,20]$.

\section{Inflammation}

Mastitis and/or abscesses are relatively common in breastfeeding women and can progress rapidly. Mastitis is most frequently caused by $S$. aureus from the nursing baby and should be treated with antibiotic therapy. Mastitis for which treatment is delayed or inadequate can progress to abscess formation. For patients who fail antibiotic treatment or in whom abscess is suspected, breast ultrasound is recommended. Mammogram is not usually needed unless malignancy is suspected. The ultrasound appearance of mastitis includes increased parenchymal echogenicity, thickening/edema of the skin, increased vascular flow on Doppler imaging, dilated lymphatics, and enlarged reactive axillary/intramammary lymph nodes. A breast abscess usually appears as a round or irregular hypoechoic (homogenous or heterogeneous) fluid collection, often with fluid-debris levels or mobile debris on real-time imaging. There may be posterior acoustic enhancement and increased peripheral vascularity.

If breast ultrasound demonstrates findings consistent with breast abscess, ultrasound-guided needle aspiration can be performed, both for therapeutic and diagnostic measures to obtain an aspirate for culture and sensitivity. If the abscess persists on follow-up ultrasound imaging, the procedure can be repeated until the abscess is no longer visible and the patient is asymptomatic. If the abscess is not amenable to needle aspiration, surgical incision and drainage may be necessary to treat the patient $[8,11,17,20-22]$.

Granulomatous mastitis is an uncommon inflammatory disease closely associated with pregnancy and lactation. It typically occurs in young women within 5 years of pregnancy [8]. It is a diagnosis of exclusion, with a relatively good prognosis, although its imaging appearance most frequently resembles malignancy. Clinically, it can present as a firm or hard mass typically sparing the subareolar breast and can have associated reactive lymphadenopathy. Corticotherapy is usually effective. Because of an association with Corynebacterium, penicillin and tetracycline should be effective if the bacteria are isolated in microbiology or pathology $[8,11]$.

\section{Nipple-Areolar Complex}

Disorders of the nipple areolar complex can occur during pregnancy, lactation, or after breastfeeding. Benign processes that may affect the nipple-areolar complex include nipple inversion related to scarring or constriction of the milk ducts 
after breastfeeding, eczema, duct ectasia, subareolar abscess, nipple adenomas, and papillomas. Malignant processes include invasive or noninvasive breast cancer, Paget's disease, and lymphoma. Patients may present with nipple inversion, retraction or enlargement, a palpable mass, nipple discharge, skin changes in and around the nipple, infection with resultant nipple changes or a subareolar mass, or abnormal findings at routine mammographic screening. Physical examination by the patient's primary care physician, dermatologist, or surgeon should be part of the diagnostic workup. Skin changes, especially if persistent after trial of appropriate therapy with moisturizers or antibiotics depending on the clinical scenario, should be sampled with a skin punch biopsy or nipple wedge resection to exclude Paget's disease. The evaluation and management of these symptoms is the same as in the nonpregnant patient. Disorders of the nipple-areolar complex require a diagnostically specific imaging evaluation beginning with high-frequency transducer sonography.

\section{Nipple Discharge}

Bloody nipple discharge is present in up to $20 \%$ of pregnant patients and up to $15 \%$ of lactating individuals [8, 23, 24]. Knowledge of what constitutes abnormal nipple discharge is critical in the appropriate management of this frequent symptom. Spontaneous, clear, bloody, or serous nipple discharge is considered pathologic. Spontaneous nipple discharge constitutes nearly $7 \%$ of all breast complaints [25]. In the lactating patient, true bloody nipple discharge should be distinguished from cracked or bleeding nipples related to nursing.

Bloody nipple discharge can be physiologic during pregnancy occurring most frequently during the third trimester when the vascularity of the breast is highest. Bilateral nipple discharge, discharge arising from multiple duct orifices, or discharge of white or green color is less suspicious. Other etiologies include hypothyroidism, pituitary adenomas, and side effects related to certain classes of medications, such as antiemetics, antihypertensives, psychotropics, hormonal drugs, and H2-receptor antagonists. This type of discharge should be managed clinically and imaging often has no significant role.

Pathologic nipple discharge, from either benign or malignant causes, is usually unilateral and arises from one duct orifice; $1 \%$ to $45 \%$ of women presenting with pathologic nipple discharge will have breast cancer, usually invasive ductal carcinoma [26]. Furthermore, nipple discharge may be the only sign of malignancy. However, the most common mass associated with pathologic nipple discharge is a benign intraductal papilloma.

First-line management in the pregnant patient with bloody nipple discharge is a high-frequency ultrasound of the subareolar region to search for an intraductal mass.
Doppler can be helpful to detect vascularity within an intraductal mass, but the lack of vascularity does not exclude malignancy. Mammography should be considered for further evaluation, but its definitive role in the pregnant patient is not known. In the nonpregnant patient, a mammogram will be positive in $50 \%$ to $90 \%$ of malignancies [27].

MR ductography uses a heavily weighted $\mathrm{T} 2$ sequences, does not require contrast, is noninvasive, and does not use any radiation. MR ductography will demonstrate dilated ducts and show ductal masses as signal defects [28]. Noncontrast MR is not helpful in determining if an intraductal mass is benign or malignant and MR ductography is not widely available at most institutions. In general, for persistent pathologic nipple discharge, if an intraductal mass is not identified on ultrasound or at mammography, the patient should be referred to a breast surgeon for possible surgical duct excision.

\section{Imaging Workup}

\section{Ultrasound}

Real-time, gray-scale and color Doppler sonography with a linear, high-frequency transducer is the initial step in the imaging evaluation of symptomatic patients pregnant or nonpregnant who are younger than age 30 years. It is noninvasive and highly sensitive (nearly $100 \%$ ) and does not expose the patient to ionizing radiation [8]. Recent data suggest that ultrasound also may be the first line of imaging for all women younger than age 40 years [29].

On review of 22 cases of PABC, Ahn et al. reported a slightly higher sensitivity of ultrasound (100\%) compared with mammography (87\%) [3]. Robbins et al. assessed the accuracies of both mammogram and ultrasound in their review of 134 lesions detected during pregnancy or postpartum period. Individually, the sensitivity of mammography and sonography was $100 \%$. The specificity of each was $93 \%$ and $86 \%$, the positive predictive values were $40 \%$ and $19 \%$, and the negative predictive values were $100 \%$ and $100 \%$ respectively.

\section{Mammography}

Radiation is an important consideration in the pregnant patient because of concerns about exposure to both the mother and fetus. With current digital mammography techniques, the mean average glandular dose from a bilateral two-view digital mammogram is $3.7 \mathrm{mGy}$ compared with 4.74 mGy ( $22 \%$ decrease per acquired view) from filmscreen mammography.

Many of the articles reporting on the radiation dose of mammography to the fetus have estimated fetal dose ranging from 0.01 Gy to $0.004 \mathrm{~Gy}[8,9]$, far less than the radiation dose 
of $0.05 \mathrm{~Gy}$ that is estimated to induce fetal malformations [30]. Based on this information, the authors conclude that mammography during pregnancy is safe, yet should be avoided during the first trimester when organogenesis occurs $[2,8]$.

Subsequent articles report the dose to the fetus from a digital mammogram with proper abdominal shielding to be even less and therefore negligible [31]. Sechopoulos et al. prospectively determined the radiation dose to the organs of the body, including the uterus from a bilateral two-view mammogram using Monte Carlo simulations and a phantom. The simulation used clinically relevant $\mathrm{x}$-ray energies and the effect from using abdominal shielding (thickness of $0.25 \mathrm{~mm}$ resulting in absorption of $99.7 \%$ of x-rays of $35 \mathrm{keV}$ ) also was investigated. He concluded that the radiation dose to all tissues other than the breast is extremely low and that, specifically, the dose to the first-trimester fetus is minimal [32]. Counseling the pregnant women before mammography may help to alleviate concerns and has been covered in detail in a recent review article [33].

Another consideration to be given to mammography is the radiographic density of the breast tissue during pregnancy. However, it should be noted that not all pregnant or lactating women demonstrate a significant increase in parenchymal density above baseline [9]. Furthermore, mammographic breast density in the lactating woman can be decreased by imaging directly after nursing. Mammography is superior to ultrasound in detecting and evaluating microcalcifications as well as architectural distortion. Many studies have demonstrated the ability of mammography to detect cancer despite dense breast tissue [2, 8, 9]. Yang et al. in a retrospective review of 20 pregnant patients imaged preoperatively found the sensitivity of mammography to be $90 \%$ for detecting suspicious features of malignancy. Thirty-three percent of these tumors exhibited secondary features of malignancy, considered to be more subtle and felt to contribute to the false-negative rate associated with mammography during pregnancy [9].

\section{Magnetic Resonance Imaging}

Breast magnetic resonance imaging (MRI) is generally not considered a useful diagnostic tool in pregnant and lactating patients for several reasons. First, intravenous gadolinium is administered as a contrast agent in these studies. Although very little of this agent crosses the placenta [34], it is a class $\mathrm{C}$ drug and most institutions will not use it unless the benefit outweighs the risk for the mother's health. Second, because of the rapid enhancement of lactating breast parenchyma with gadolinium, the detection of breast cancer is significantly limited [8]. Last, there is concern about a breastfed baby's exposure to gadolinium. Previous guidelines indicate that the mother should discontinue breastfeeding or discard pumped breast milk for 24 hours following its administration. However, some conclude that mothers receiving gadolinium contrast can safely breast feed without cessation [34] because of the very small amount of gadolinium excreted in breast milk (less than $0.1 \%$ of the dose administered to the mother), the very small amount of gadolinium that enters the gut of the breastfed infant $(<1 \%$ of the recommended IV dose for an infant), and the very small amount absorbed from the gut by the infant.

\section{Image-Guided Procedures}

The effect of pregnancy on benign lesions and carcinomas results in significant overlap in their imaging appearances. Due to this overlap, distinguishing benign lesions from breast cancer can be difficult and biopsy may be indicated. In general, core needle biopsy, fine needle aspiration, and excisional surgical biopsy during pregnancy and lactation are safe. However, ultrasound-guided core biopsies offer additional advantages with minimal risk to the patient or the fetus. It provides adequate specimens for confident diagnosis and further evaluation with staining or immunohistochemical assays and yields low false-positive results with an accuracy of $99 \%$. Because of hyperplastic changes with atypia during pregnancy, fine aspiration can produce false-positive results and thus the interpretation should be made by a skilled pathologist informed of the patient's pregnant or lactating status. Furthermore, ultrasoundguided biopsy eliminates risks associated with surgery and anesthesia and is less expensive.

Patients should be counseled of the potential risks and benefits of core biopsy. Risks include: infection, hematoma, bloody nipple discharge shortly after biopsy, failure to obtain a diagnostic specimen, pneumothorax, and a rare complication that is unique to the lactating patient, known as a milk fistula. The risk of infection after core biopsy is extremely low with proper aseptic technique. Because of increases in vascularity to the breasts during pregnancy and lactation, there may be slightly more bleeding during the biopsy than in nonpregnant patients. If the suspicious abnormality is located near the nipple, patients may experience bloody nipple discharge for several days following the biopsy. The potential complication of a milk fistula using this biopsy technique has been case reported but is extremely uncommon [35]. This complication is more common following open surgical biopsies. Although not life-threatening, a milk fistula can be an annoyance to the patient and difficult to manage. The fistula may dry up spontaneously or resolve only after the cessation of breastfeeding. These small risks should not prevent biopsy of a suspicious abnormality.

\section{Conclusions}

The majority of breast disorders that occur in nonpregnant patients also occur in pregnant patients, including breast 
malignancy. Pregnancy-associated breast cancer is a rare but important phenomenon, because there is the potential for considerable delay in diagnosis and treatment in the pregnant or lactating patient. Although breast cancer is uncommon in pregnancy, obstetric providers should be familiar with evaluating presenting signs and symptoms to minimize this delay. All breast symptoms in the pregnant patient, but particularly palpable masses, should be carefully evaluated with physical examination and initial diagnostic ultrasound. Mammography with abdominal shielding may safely be used throughout pregnancy without harm to the fetus but may be reserved for evaluation of suspicious lesions detected at initial diagnostic ultrasound.

Disclosures No potential conflicts of interest relevant to this article were reported.

\section{References}

1. American College of Obstetricians and Gynecologists. Practice bulletin No. 122. Obstet Gynecol. 2011;118:372-82.

2. Robbins J, Jeffries D, Roubidoux M, Helvie M. Accuracy of diagnostic mammography and breast ultrasound during pregnancy and lactation. AJR Am J Roentgenol. 2011;196:716-22.

3. Ahn BY, Kim HH, Moon WK, et al. Pregnancy and lactationassociated breast cancer: mammographic and sonographic findings. J Ultrasound Med. 2003;22:491-7.

4. Saunders CM, Baum M. Breast cancer and pregnancy: a review. JR Soc Med. 1993;86:162-5.

5. Andersson T, Johansson A, Chung-Cheng H, et al. Increasing incidence of pregnancy-associated breast cancer in Sweden. Obstet Gynecol. 2009;114:568-72.

6. Ayappan A, Kulkarni S, Crystal P. Pregnancy-associated breast cancer: spectrum of imaging appearances. Br J Radiol. 2010;83:529-34.

7. Buré LA, Azoulay L, Benjamin A, Abenhaim HA. Pregnancyassociated breast cancer: a review for the obstetrical care provider. J Obstet Gynaecol Can. 2011;33(4):330-7. Review.

8. Sabate JM, Clotet M. Torrubia S, et al. Radiographic evaluation of breast disorders related to pregnancy and lactation. RadioGraphics. 2007;27(Spec Issue):S101-S124.

9. Yang WT, Dryden MJ, Gwyn K, Whitman GJ, Theriault RL. Imaging of breast cancer diagnosed and treated with chemotherapy during pregnancy. Radiology. 2006;239:52-60.

10. Hahn K, Johnson PH, Gordon N, Kuerer H, Middleton L, Ramirez M, Yang W, Perkins G, Hortobagyi GN, Theriault RL. Treatment of pregnant breast cancer patients and outcomes of children exposed to chemotherapy in utero. Cancer. 2006;107(6):1219-26.

11. Ikeda DM. Mammographic and ultrasound analysis of breast masses. In: Thrall JH, editor. The requisites: breast imaging. Philadelphia: Elsevier Mosby; 2004. p. 90-132.

12. Greenberg R, Skornick Y, Kaplan O. Management of breast fibroadenomas. J Gen Intern Med. 1998;13:640-5.
13. Graf O, Helbich TH, Hopf G, Graf C, Sickles EA. Probably benign breast masses at US: is follow-up an acceptable alternative to biopsy? Radiology. 2007;244:87-93.

14. Harvey JA, Nicholson BT, Lorusso AP, Cohen MA, Bovbjerg VE. Short-term follow-up of palpable breast lesions with benign imaging features: evaluation of 375 lesions in 320 women. AJR Am J Roentgenol. 2009;193:1723-30.

15. Baker TP, Lenert JT, Parker J, et al. Lactating adenoma: a diagnosis of exclusion. Breast J. 2001;7:354-7.

16. Behrndt VS, Barbakoff D, Askin FB, Brem RF. Infarcted lactating adenoma presenting as a rapidly enlarging breast mass. AJR Am J Roentgenol. 1999;173:933-5.

17. Hogge JP, Shaw de Paredes E, Magnant CM, Lage J. Imaging and management of breast masses during pregnancy and lactation. Breast J. 1999;5:272-83.

18. Way JC, Culham BA. Phyllodes tumor in pregnancy: a case report. Can J Surg. 1998;4:407-9.

19. Vorherr H, Vorherr U, Kutvirt D, et al. Cystosarcoma phyllodes: epidemiology, pathohistology, pathobiology, diagnosis, therapy and survival. Arch Gynecol. 1985;236(3):173-81.

20. Son EJ, Oh KK, Kim EK. Pregnancy-associated breast disease: radiologic features and diagnostic dilemmas. Yonsei Med J. 2006;47:34-42.

21. Ulitzsch D, Nyman MK, Carlson RA. Breast abscess in lactating women: US-guided treatment. Radiology. 2004;232:904-9.

22. Eryilmaz R, Sahin M, Tekelioglu MH, Daldal E. Management of lactational breast abscesses. Breast. 2005;14:375-9.

23. Lafreniere R. Bloody nipple discharge during pregnancy: a rationale for conservative treatment. J Surg Oncol. 1990;43:228-30.

24. Kline TS, Lash SR. The bleeding nipple of pregnancy and postpartum: a cytologic and histologic study. Acta Cytol. 1964;8:336-40.

25. Sauter ER, Schlatter L, Lininger J, Hewett JE. The association of bloody nipple discharge with breast pathology. Surgery. 2004;136:780-5.

26. Sickles EA. Galactography and other imaging investigations of nipple discharge. Lancet. 2000;356:1622-3.

27. Koskela A, Berg M. Breast lesions causing nipple discharge: preoperative galactography-aided stereotactic wire localization. AJR Am J Roentgenol. 2005;184:1975-8.

28. Masonori H, Hiroshi N, Takehiko G. MR ductography: comparsion with conventional ductography as a diagnostic method in patients with nipple discharge. Radiographics. 2007;27:S183-96.

29. Lehman CD, Lee CI, Loving V, Portillo MS, Peacock S, DeMartini WB. Accuracy and value of breast ultrasound for primary imaging evaluation of symptomatic women 30-39 years of age. AJR Am J Roentgenol. 2012;199:1169-77.

30. Greskovich Jr J, Macklis R. Radiation therapy in pregnancy: risk calculation and risk minimization. Semin Oncol. 2000;27:633-45.

31. Behrman RH, Homer MJ. Mammography and fetal dose. Radiology. 2007;243:605-6.

32. Sechopoulos I, Suryanarayanan S, Vedantham S, D'Orsi C, et al. Radiation dose to organs and tissues from mammography: Monte Carlo and phantom study. Radiology. 2008;246:434-43.

33. Brent RL. Saving lives and changing family histories: appropriate counseling of pregnant women and men and women of reproductive age, concerning the risk of diagnostic radiation exposures during and before pregnancy. Am J Obstet Gynecol. 2009;200 (1):4-24. doi:10.1016/j.ajog.2008.06.032.

34. Webb JA, Thomsen HS, Morcos SK. The use of iodinated and gadolinium contrast media during pregnancy and lactation. Eur Radiol. 2005; 15:1234-40.

35. Schackmuth E, Harlow C, Norton L. Milk fistula: a complication after core breast biopsy. AJR Am J Roentgenol. 1993;961-962. 